DR PASWACH WIRIYAKIJJA (Orcid ID : 0000-0002-2340-517X)

DR STEFANO FEDELE (Orcid ID : 0000-0001-9006-9412)

Article type : Original Article

\title{
Development and validation of a short version of Chronic Oral Mucosal Disease Questionnaire (COMDQ-15)
}

\author{
Paswach Wiriyakijja ${ }^{1,2 *}$, Stephen Porter ${ }^{1}$, Stefano Fedele ${ }^{1,3}$, Tim Hodgson ${ }^{4}$, Roddy \\ McMillan $^{4}$, Martina Shephard ${ }^{4}$, Richeal Ni Riordain ${ }^{1,5}$ \\ ${ }^{1}$ UCL Eastman Dental Institute, London, United Kingdom \\ ${ }^{2}$ Department of Oral Medicine, Faculty of Dentistry, Chulalongkorn University, Bangkok, Thailand \\ ${ }_{3}^{3}$ NIHR University College London Hospitals Biomedical Research Centre, London, United Kingdom \\ ${ }^{4}$ Eastman Dental Hospital, UCLH Foundation NHS Trust London, London, United Kingdom \\ ${ }^{5}$ Department of Oral Medicine, Cork University Dental School and Hospital, Cork, Ireland. \\ ${ }^{*}$ Corresponding author. \\ E-mail address: paswach.w@gmail.com
}

Running title: Short-form Chronic Oral Mucosal Disease Questionnaire

Key words: Quality of Life; Chronic oral mucosal disease; Short form

This article has been accepted for publication and undergone full peer review but has not been through the copyediting, typesetting, pagination and proofreading process, which may lead to differences between this version and the Version of Record. Please cite this article as doi: 10.1111/JOP.12964

This article is protected by copyright. All rights reserved 


\section{ABSTRACT}

Background: The adoption of the Chronic Oral Mucosal Disease Questionnaire (COMDQ) into clinical practice has been low, despite its rigorous development process. A potential limitation of the COMDQ is the high response burden to patients. Therefore, the aim of the present study was to develop and validate a short version of the 26-item COMDQ.

Methods: The COMDQ data of 520 patients with chronic oral mucosal diseases were randomly divided into 2 subsamples. Descriptive item analysis and exploratory factor analysis (EFA) were performed using data from the first subsample for item reduction and development of the shortened COMDQ. The resulting short version was then validated using confirmatory factor analysis (CFA) on the other subsample. Internal consistency reliability of the short-form COMDQ was assessed using Cronbach's alpha. Criterion validity of this new scale was examined against its original version.

Results: Based upon item analysis, 11 items were dropped. EFA results on the remaining 15 items extracted 4 factors consistent with the original COMDQ, and CFA results displayed acceptable goodness-of-fit indices of this factor structure on different sample. The COMDQ-15 was then created. Cronbach's alpha of 4 subscale scores ranged from 0.7 to 0.91 , indicating good internal consistency reliability of the COMDQ-15. Correlations between total and subscale scores of the COMDQ-15 and its parent scale were high, supporting good criterion validity of this shortened scale.

Conclusion: The COMDQ-15 is a brief, valid and reliable instrument that can give an overview of the patient's quality of life related to their chronic oral mucosal conditions. 


\section{Introduction}

Chronic oral mucosal disorders constitute a heterogeneous spectrum of inflammatory conditions causing a variety of oral manifestations, which are persistent and/or recurrent over time. Inflammatory and ulcerative lesions associated with these conditions typically cause a variable degree of symptoms ranging from mild discomfort to severe debilitating pain, which can compromise normal oral functioning and have a significant impact on the psychosocial well-being and overall quality of life (QoL) of the affected individuals ${ }^{1}$. The management of these conditions may also require the administration of topical or systemic medications, including the use of corticosteroids and immunosuppressant agents, which can cause adverse effects and also impair patients' $\mathrm{QoL}^{2}$. It is therefore appropriate and advisable that the assessment of therapeutic interventions in individuals with chronic oral mucosal disorders should include QoL measures ${ }^{3}$. From the clinician's perspective, improvement of QoL is considered to be ultimate therapeutic goal for patients affected by these conditions ${ }^{4}$. In addition, the use of QoL measures could help identifying patient preference for specific interventions, as well as facilitating communication with patients in clinical practice ${ }^{5}$.

The Chronic Oral Mucosal Disease Questionnaire (COMDQ) is a self-reported questionnaire assessing QoL in individuals with chronic oral mucosal diseases including oral lichen planus, recurrent aphthous stomatitis, pemphigus vulgaris and mucous membrane pemphigoid6. This self-administered scale has been proven to be psychometrically sufficient in a series of validation studies conducted in several countries $^{7-11}$. The COMDQ was found to have good level of content validity owing to incorporation of patient's views and preferences during its development process ${ }^{6}$. The original version of the COMDQ comprises 26 items capturing 4 domains including pain and functional limitation, medication and treatment, social and emotional, and patient support. The COMDQ, however, appears to be under-implemented in both clinical research and routine Oral Medicine practice despite its indicated need and utility. This might be related to time needed to complete all 26 items of the questionnaires (high response burden to patients), which can conflict with the current time constraints of the healthcare service ${ }^{12}$. We suggest that the development of a shorter version of COMDQ with optimal balance between its brevity, key content coverage and psychometric 
performance could improve widespread adoption into clinical practice. Thus, the aim of the present study was to develop the short version of the COMDQ without altering the dimensional structure and psychometric quality.

\section{Methods}

\section{Study design}

This was a development and validation study using baseline data from the Determination of Minimal Important Difference and Patient Acceptable Symptom State of Patient Reported Outcome Measures in Immunologically mediated Oral Mucosal Diseases (MEAN-IT) study, which had favourable opinion from the London - Queen Square Research Ethics Committee (REC reference 17/LO/1825; approval date 3 November 2017).

\section{Participants}

From January 2018 to August 2019, a convenient sample of 520 patients with chronic oral mucosal conditions including oral lichen planus (OLP), recurrent aphthous stomatitis (RAS), pemphigus vulgaris (PV) and mucous membrane pemphigoid (MMP) was recruited from the Oral Medicine clinic, UCLH Eastman Dental Hospital, London, United Kingdom. All potentially eligible participants, in all Consultant lead Oral Medicine clinics were invited to participate (conducted by PW). The inclusion and exclusion criteria of study participants are listed in Table 1. Patient participation was voluntary, and the data were handled anonymously.

\section{Sample size}

For robust psychometric evaluation to be performed, the numerical ratio between respondents and items should be at least 10:1 for conducting factor analyses ${ }^{13}$. As two different types of factor analyses, namely exploratory factor analysis (EFA) and confirmatory factor analysis (CFA), were employed for development and validation process of the short version of the 26-item COMDQ, a total number of 520 participants were required for the present study.

\section{Outcome measures}


The COMDQ comprises 26 items in four subscales including Pain and Functional limitation (PF, 9 items), Medication and treatment (MT, 6 items), Social and Emotional (SE, 7 items) and Patient Support (PS, 4 items). The items were answered on a 5-point Likert-type scale (0-4), ranging from "not at all" to "extremely". Total COMDQ score is calculated by summation of the responses of all items, giving the possible maximum score of $104^{6}$.

\section{Procedures}

The COMDQ data of 520 participants of the MEAN-IT study were extracted for the present study. In addition, the following demographic and clinical data were collected for the purpose of sample descriptions and contrasted group comparisons: age, gender, ethnicity and clinical types of OLP (reticular/plaque, atrophic/erosive, ulcerative) and RAS (minor, major, herpetiform). The COMDQ items and subscales were initially analysed using descriptive statistics for preliminary item reduction. The cross-sectional samples of the MEAN-IT study were randomly split into two approximately equal datasets $(\mathrm{N}=260)$, namely "development sample" and "validation sample". The COMDQ data from the development sample were analysed using EFA to identify underlying factor (subscale) of the COMDQ and associated items in each factor, and the results were used as further evidence for item reduction and generation of the short-versioned COMDQ. To validate short-form COMDQ, CFA was performed to test the hypothesized factor structure of this brief COMDQ determined from the EFA with an independent validation sample. Reliability and validity of new scale were also compared with its original version.

\section{Statistical analyses}

Statistical analyses were performed using MPlus version 8.2 (Muthén \& Muthén, 2015) and STATA version 15.1 (StataCorp, College Station, TX, U.S.A.). Descriptive demographic and clinical characteristics of the sample were first summarised using mean, standard deviation and proportion. Descriptive Item statistics including mean, standard deviations, floor and ceiling effects (proportion of item endorsement at the lowest and highest response options) were calculated. For preliminary item reduction process, items with floor effects of $\geq 60 \%$ suggesting less relevant items were first eliminated. Next, adjusted item-total correlations were calculated, and an item with low 
correlation $(<0.3)$ was considered discarded due to poor metric performance compared to the remainder of the scale. Then a matrix of inter-item polychoric correlations was constructed, and one item from each of item pairs with high correlations (>0.7) was considered deleted to minimize information redundancy.

EFA using weighted least square means and variance-adjusted (WLSMV) estimator and oblique rotation (Promax) was carried out on the development sample. The WLSMV estimator is appropriate for the ordered categorical nature of the COMDQ data, and oblique rotations allow for correlations between underlying factors ${ }^{14}$. The optimal number of factor extraction was based upon eigenvalues $\geq 1$, further inspection of the corresponding scree plot (number of dots above the elbow of the plot where the notable decline in factors levels off), and factor interpretability according to item content within each extracted factor. Items retention was based upon at least 0.4 loadings on a certain factor. For the item reduction, Item were considered removed if they failed to load with sufficient strength $(<.03)$ on any factor or had high cross-loading $(>0.3)^{15}$.

With the remaining half of the data (validation sample), a CFA was performed to determine whether identified factor structure could be replicated on different sample. To confirm model fit, several fit indices including root mean square of error approximation (RMSEA), standardized root mean squared residual (SRMR), comparative fit index (CFI) and Tucker-Lewis index (TLI) were calculated. RMSEA and SRMR values closer to 0 indicate better fit, with values below 0.08 and 0.05 indicating acceptable and good fit, respectively. CFI and TLI values greater than 0.95 are considered acceptable ${ }^{16}$. For measures of internal consistency reliability, Cronbach's alpha coefficient $(\alpha)$ for each subscale was computed, and a reliability value of 0.70 or above indicates good reliability of the scale ${ }^{17}$. Criterion validity of the short-form COMDQ was evaluated by assessing the strength of the correlations between subscale scores of the short and original version of the COMDQ. The primary hypotheses were that scores of short-version COMDQ would be significantly and positively correlated with scores of its original scale.

\section{Results}

Sample characteristics 
Study sample consisted of 520 participants with chronic oral mucosal diseases including 306 patients with OLP, 130 patients with RAS, 33 patients with PV and 51 patients with MMP. The average age of the participants was 58.39 years and $71.73 \%$ were female. The majority of sample $(71.35 \%)$ were Caucasians, followed by $22.31 \%$ Asians, $3.85 \%$ Blacks and $2.5 \%$ mixed ethnic groups. In comparison with other conditions, patients with RAS reported highest mean COMDQ scores $(47.31 \pm 16.35)$ indicating the worst oral health-related quality of life, followed by PV (42.73 \pm 17.91$)$, and OLP (39.38 \pm 19.40). The sample was randomly split into two subsamples, and Table 2 summarised descriptive characteristics of two random samples, and both were similar in all variables.

\section{Item and subscale analyses of the original COMDQ}

Individual item analyses including mean, standard deviation, floor and ceiling effects using the whole sample are listed in Table 3. Item PF9 (discomfort/denture) was dropped in this stage due to its floor effect of $>90 \%$, suggesting low impact of this item on the vast majority of respondents. The following correlation analyses for the remaining 25 items involved the development sample only. Four out of the 25 items had adjusted item-total correlations below 0.3 (Table 2). Item MT2 (medication satisfaction) and PS1 (satisfaction on available information) were discarded while item PS2 (support from family) and PS3 (support from friends/colleagues) were retained, as they were felt to represent distinct domain of "patient support" consistent to a conceptual framework of the original COMDQ.

Further inspection of inter-item polychoric correlation matrix revealed 18 item pairs with correlations over 0.7 , indicating content redundancy, and inclusion of both items in the pair are unnecessary. Dropping item PF2 (limitation/food types), PF4 (limitation/food texture), PF6 (limitation/food temperature), PF8 (limitation/oral hygiene care), MT6 (frustration on no disease cure), SE3 (stress due to oral condition), SE5 (worry about the future), and PS4 (isolation due to oral disease) eliminated 14 of these 18 strong inter-item correlations.

\section{Exploratory factor analysis}

Exploratory factor analysis using Promax rotation on the remaining 15 items yielded 4 factors with eigenvalues greater than 1 (Table 4), and this was further confirmed by the corresponding scree plot. All the items had factor loadings over 0.3 on their designated 
factors except for item MT1 (medication need), which was moved to the original Pain and Functional limitation subscale. No cross-loading was observed, and therefore no items met criteria for elimination at this stage. The new 15-item version of the COMDQ (COMDQ-15) was then created (Supplementary 1). Three factors (Medication and Treatment, Social and Emotional, Patient Support) were named according to the original scale while the original "Pain and Functional limitation" factor was changed to "Physical Discomfort" to better reflect content of the remaining items within this factor. This new 4factor solution of the 15-item COMDQ served as the hypothesized model for the subsequent CFA.

\section{Confirmatory factor analysis}

Confirmatory factor analysis was performed to test structural validity of the COMDQ-15 by replicating hypothesized model identified by EFA in validation sample $(\mathrm{N}=260)$. The goodness-of-fit indicators for the 4-factor solution of the COMDQ-15 compared to its original COMDQ-26 were reported in Table 5. CFA results of the COMDQ-15 demonstrated acceptable level of RMSEA and satisfactory level of the remaining fit indices; whereas, the original 26-item COMDQ was found to have insufficient level of structural validity based upon expected fit indices.

\section{Internal consistency reliability and criterion validity}

The estimated values of Cronbach's alpha for 4 subscales of the COMDQ-15 were as followed: 0.86 for "Physical Discomfort", 0.71 for "Medication \& Treatment", 0.91 for "Social \& Emotional" and 0.70 for "Patient Support". Overall, the reliability coefficients indicated acceptable to good level of internal consistency reliability of the short version of the COMDQ. Criterion validity of the COMDQ-15 was satisfactory as both total and subscale scores of the short and original version of COMDQ were significantly and highly correlated $\left(r_{\mathrm{s}}\right.$ range $=0.88-0.99$; see also Table 6$)$.

\section{Discussion}

The present study reports the development and initial validation of a 15-item brief version of the Chronic Oral Mucosal Disease Questionnaire, which retains content coverage of QoL related to chronic oral mucosal conditions from its original scale. In accordance with 
classical test theory requirements, item analysis, structural validity, internal consistency reliability and criterion validity were studied to ensure that this short version maintains the psychometric quality of its full-length scale. Items with low functionality and conformity to the whole scale or those with information-redundant were removed to refine and create the most economical scale.

Content validity of the COMDQ-15 was inherited from the patient-centred qualitative study during the development of its original version ${ }^{6}$, and was ascertained by an attempt to preserve all the relevant aspects of hypothesized QoL construct during item reduction process. The underlying four theoretical subscales of the COMDQ-15 were identified by exploratory factor analysis and the stability of this factor structure was confirmed in a replication sample. The original item MT2 "medication need" was moved to the Physical Discomfort subscale, which appeared conceptually sensible considering greater level of physical discomfort generally increase the need for medication. Despite considerable shortening of its full-length scale, the COMDQ-15 had good to excellent level of internal consistency reliability and its subscales were significantly and strongly correlated with each corresponding original subscales $\left(r_{s} \geq 0.88\right)$, indicating that this 15 -item version appeared to be a valid and reliable summary of its original scale.

The notable advantage of having a short-form COMDQ is the lower respondent burden, making it easier to administer and thereby providing a more practical scale for use in routine clinical settings. Not only could shortened outcome measures increase patient acceptability in daily practice, but they could also enhance feasibility in clinical trials and other clinical studies. One example is the extensive usage of the shortened 14-item Oral Health Impact Profile (OHIP-14) $)^{18}$ in oral mucosal disease literature. Two recent reviews found significantly higher frequency of use of the OHIP-14 than its original lengthy version (OHIP-49) as outcome measures in previous research of OLP (12 times use of the OHIP-14 compared to 6 for the OHIP-49) and RAS (9 times use of OHIP-14 compared to one study for the OHIP-49) ${ }^{19,20}$. Considering the importance of measuring patient's QoL in oral mucosal diseases, the development of COMDQ-15 could improve implementation of this instrument in both clinical and research settings.

The present study has a number of limitations. Shortening questionnaires is always a trade-off between resources (e.g. time and cost) saved and the amount of information 
lost. Information concerning oral functional limitation (PF2, PF4, PF6, PF8) and patient satisfaction (MT2, PS1) were present in the original 26-item COMDQ but are no longer represented in the new shortened version. Clinicians and researchers who are interested in capturing these data should refer to the original COMDQ, which remains a valid and comprehensive measure of QoL in chronic oral mucosal conditions. In addition, although the present shortened scale appears to be psychometrically sound, it still requires additional psychometric testing particularly on sensitivity to change and interpretability of its score.

\section{Conclusions}

The COMDQ-15 is a brief, easy-to-use, valid and reliable instrument that can give an overview of the patient's perspective on QoL related to their chronic oral mucosal conditions. Although additional psychometric testing is needed to confirm sensitivity to change and interpretability of its score, the COMDQ-15 shows notable potential to assist clinicians in daily practice, so to assess the burden of chronic oral mucosal conditions upon QoL and measure relevant changes after medical intervention. It can also be easily adopted in clinical trials and other clinical studies. This marks another significant step towards the accurate and methodologically valid measurement of QoL in individuals with chronic oral mucosal diseases. It also highlights the importance of incorporating patients' views and perception into clinical decision making, so improving the quality of patient care in Oral Medicine.

\section{Author contributions}

Paswach Wiriyakijja and Richeal Ni Riordain designed the study. Paswach Wiriyakijja collected data from patients, carried out the statistical analyses reported in the study and drafted manuscript. Richeal Ni Riordain, Roddy McMillan, Martina Shephard, Tim Hodgson, Stefano Fedele and Stephen Porter edited and contributed comments on the manuscripts.

\section{Acknowledgements}

Paswach Wiriyakijja would like to thank Dr Emma Hayes, Dr Priya Thakrar, Dr Krupali Patel, Dr Barbara Carey, Dr Carolina Venda Nova, Dr Craig Whitelaw, Dr Sanjeet 
Singhota, Dr Thomas Saunsbury and Dr Valeria Mercadante for their substantial help and supports towards the recruitment process of the study. Paswach Wiriyakijja received a PhD Scholarship from the Faculty of Dentistry, Chulalongkorn University, Bangkok, Thailand. Stefano Fedele received funding from the National Institute for Health Research University College London Hospitals Biomedical Research Centre. The MEANIT study received service support funding from the National Institute for Health Research University College London Hospitals Biomedical Research Centre and the NIHR Clinical Research Network Portfolio.

\section{Disclosure statement}

No potential conflict of interest was reported by the authors. 


\section{References}

1. Ni Riordain R, Wiriyakijja P. Patient reported outcome and experience measures of oral disease in oral medicine. British dental journal. 2017;223(9):713.

2. Mustafa MB, Porter SR, Smoller BR, Sitaru C. Oral mucosal manifestations of autoimmune skin diseases. Autoimmunity reviews. 2015;14(10):930-951.

3. Skevington SM. Investigating the relationship between pain and discomfort and quality of life, using the WHOQOL. Pain. 1998;76(3):395-406.

4. Davari P, Hsiao HH, Fazel N. Mucosal lichen planus: an evidence-based treatment update. American journal of clinical dermatology. 2014;15(3):181-195.

5. Chen J, Ou L, Hollis SJ. A systematic review of the impact of routine collection of patient reported outcome measures on patients, providers and health organisations in an oncologic setting. $B M C$ health services research. 2013;13:211.

6. Ni Riordain R, Meaney S, McCreary C. A patient-centered approach to developing a quality-of-life questionnaire for chronic oral mucosal diseases. Oral surgery, oral medicine, oral pathology, oral radiology, and endodontics. 2011;111(5):578-586, 586.e571-572.

7. Ni Riordain R, McCreary C. Validity and reliability of a newly developed quality of life questionnaire for patients with chronic oral mucosal diseases. Journal of oral pathology \& medicine : official publication of the International Association of Oral Pathologists and the American Academy of Oral Pathology. 2011;40(8):604-609.

8. Ni Riordain R, McCreary C. Further reliability and responsiveness of the Chronic Oral Mucosal Diseases Questionnaire. Oral diseases. 2012;18(1):60-66.

9. Li M, He SL. Reliability and validity of the Chinese version of the chronic oral mucosal diseases questionnaire. Journal of oral pathology \& medicine : official publication of the International Association of Oral Pathologists and the American Academy of Oral Pathology. 2013;42(2):194199.

10. Ni Riordain R, Hodgson T, Porter S, Fedele S. Validity and reliability of the Chronic Oral Mucosal Diseases Questionnaire in a UK population. Journal of oral pathology \& medicine : official publication of the International Association of Oral Pathologists and the American Academy of Oral Pathology. 2016;45(8):613-616.

11. Shirzad A, Bijani A, Mehryari M, Motallebnejad M, Mohsenitavakoli S. Validity and reliability of the persian version of the chronic oral mucosal diseases questionnaire. Caspian journal of internal medicine. 2018;9(2):127-133.

This article is protected by copyright. All rights reserved 
12. Morris J, Perez D, McNoe B. The use of quality of life data in clinical practice. Qual Life Res. 1998;7(1):85-91.

13. DeVellis RF. Scale development : theory and applications. 2017.

14. Li C-H. Confirmatory factor analysis with ordinal data: Comparing robust maximum likelihood and diagonally weighted least squares. Behavior Research Methods. 2016;48(3):936-949.

15. Tabachnick BG, Fidell LS. Using multivariate statistics. Boston: Pearson Education; 2013.

16. Hu Lt, Bentler PM. Cutoff criteria for fit indexes in covariance structure analysis: Conventional criteria versus new alternatives. Structural Equation Modeling: A Multidisciplinary Journal. 1999;6(1):1-55.

17. Nunnally JC, Bernstein IH. Psychometric theory. New Delhi: Tata McGraw-Hill Ed.; 2010.

18. Slade GD. Derivation and validation of a short-form oral health impact profile. Community dentistry and oral epidemiology. 1997;25(4):284-290.

19. Wiriyakijja P, Fedele S, Porter SR, Mercadante V, Ni Riordain R. Patient-reported outcome measures in oral lichen planus: A comprehensive review of the literature with focus on psychometric properties and interpretability. Journal of oral pathology \& medicine : official publication of the International Association of Oral Pathologists and the American Academy of Oral Pathology. 2018;47(3):228-239.

20. Wiriyakijja P, Fedele S, Porter S, Mercadante V, Ni Riordain R. Patient-reported outcome measures in recurrent aphthous stomatitis: A critical assessment of quality properties. Oral diseases. 2017;23(8):1168-1179.

This article is protected by copyright. All rights reserved 
Table 1 Study eligibility criteria

\begin{tabular}{|l|l|}
\hline Inclusion criteria & Exclusion criteria \\
\hline $\begin{array}{l}\text { - Aged 18 years or older } \\
\text { - Able to understand and complete } \\
\text { questionnaires }\end{array}$ & $\begin{array}{l}\text { - Having coexisting chronic neuropathic orofacial pain, such } \\
\text { as post-traumatic trigeminal neuropathic pain, persistent } \\
\text { idiopathic facial pain or burning mouth syndrome }\end{array}$ \\
\hline $\begin{array}{l}\text { - Agree to participate and provide written } \\
\text { informed consent }\end{array}$ & $\begin{array}{l}\text { - Severe systemic disease (ASA 3 or more) and/or some } \\
\text { psychiatric conditions which might affect the participation of } \\
\text { the study such as schizophrenia }\end{array}$ \\
\hline Having one of the following conditions & \\
\hline $\begin{array}{l}\text { 1. Oral lichen planus } \\
\text { OLP based upon modified WHO } \\
\text { diagnostic criteria }\end{array}$ & $\begin{array}{l}\text { - Evidence of oral epithelial dysplasia in biopsy specimen } \\
\text { - Evidence of proven hypersensitivity to dental materials } \\
\text { - Evidence of oral lichenoid lesions associated with } \\
\text { graft-versus-host disease and systemic lupus } \\
\text { erythematosus }\end{array}$ \\
\hline 2. recurrent aphthous stomatitis & \multicolumn{2}{|l}{} \\
\hline
\end{tabular}

This article is protected by copyright. All rights reserved 
- Having recurrent oral ulceration (ulcer episodes of at least twice a year)
- Having RAS-like ulcerations associated with systemic disorders such as Behcet's disease, Sweet syndrome, Ulcerative colitis, Crohn's disease, Celiac disease, autoinflammatory syndromes, or haematological abnormalities (severe anaemia, cyclic or chronic neutropenia)

3. pemphigus vulgaris

- DIF/IIF or ELISA-proven PV

4. mucous membrane pemphigoid

- DIF/IIF or ELISA-proven MMP

Table 2 Demographic and clinical characteristics of the study sample

\begin{tabular}{|l|c|c|c|}
\hline Characteristics & $\begin{array}{c}\text { Development } \\
\text { sample }(\mathrm{N}=260)\end{array}$ & $\begin{array}{c}\text { Validation } \\
\text { sample }(\mathrm{N}=260)\end{array}$ & $\begin{array}{c}\text { P-value } \\
\text { (X 2 test or t-test) }\end{array}$ \\
\hline OLP $(\mathrm{n})$ & 154 & 152 & 0.69 \\
mean age (years) & $62.81 \pm 11.78$ & $63.34 \pm 11.46$ & 0.94 \\
gender (n, \% Female) & $120(77.92)$ & $119(78.29)$ & 0.99 \\
clinical types (n, \%) & $29(18.83)$ & $28(18.42)$ & \\
reticular/plaque & $103(66.88)$ & $103(67.76)$ & \\
atrophic/erosive & $22(14.29)$ & $21(13.82)$ & \\
ulcerative & &
\end{tabular}

This article is protected by copyright. All rights reserved 


\begin{tabular}{|l|c|c|c|} 
RAS $(\mathrm{n})$ & 63 & 67 & 0.11 \\
mean age (years) & $42.08 \pm 14.56$ & $46.21 \pm 14.87$ & 0.81 \\
gender (\% Female) & $38(60.32)$ & $39(58.21)$ & 0.99 \\
clinical types (n, \%) & & & \\
minor & $55(87.30)$ & $59(88.06)$ & \\
major & $7(11.11)$ & $7(10.45)$ & \\
herpetiform & $1(1.59)$ & $1(1.49)$ & 0.79 \\
\hline PV (n) & 18 & 15 & 1 \\
mean age (years) & $57.41 \pm 20.65$ & $55.69 \pm 15.66$ & \\
gender (\% Female) & $12(66.67)$ & $10(66.67)$ & 0.95 \\
\hline MMP $(\mathrm{n})$ & 25 & 26 & 0.61 \\
mean age (years) & $67.52 \pm 8.63$ & $67.71 \pm 11.96$ & \\
gender $(\%$ Female) & $18(72)$ & $17(65.38)$ & \\
\hline
\end{tabular}

Table 3 Descriptive item analysis of the whole sample $(\mathrm{N}=520)$ and adjusted item-total and item-subscale correlations of development sample $(\mathrm{N}=260)$

\begin{tabular}{|l|c|c|c|c|c|}
\hline Item & mean & sd & $\begin{array}{c}\text { floor } \\
\text { effect } \\
(\%)\end{array}$ & $\begin{array}{c}\text { ceiling } \\
\text { effect } \\
(\%)\end{array}$ & $\begin{array}{c}\text { adjusted item- } \\
\text { total } \\
\text { correlation }\end{array}$ \\
\hline Pain and Functional limitation & 2.41 & 1.21 & 8.27 & 20.77 & 0.5696 \\
PF1 discomfort/food types & 2.06 & 1.18 & 10.77 & 11.15 & 0.6386 \\
\hline
\end{tabular}




\begin{tabular}{|c|c|c|c|c|c|}
\hline PF3 discomfort/food texture & 2.23 & 1.22 & 11.15 & 15 & 0.6091 \\
\hline PF4 limitation/food texture & 1.93 & 1.22 & 16.15 & 8.85 & 0.6487 \\
\hline PF5 discomfort/food temperature & 1.7 & 1.23 & 20.58 & 7.88 & 0.5774 \\
\hline PF6 limitation/food temperature & 1.63 & 1.23 & 23.08 & 6.15 & 0.5684 \\
\hline PF7 discomfort/oral hygiene care & 2.02 & 1.19 & 12.31 & 10.77 & 0.6726 \\
\hline PF8 limitation/oral hygiene care & 1.42 & 1.28 & 32.5 & 6.92 & 0.6423 \\
\hline PF9 discomfort/denture & 0.21 & 0.71 & $90.38^{*}$ & 1.15 & $\mathrm{~N} / \mathrm{A}$ \\
\hline \multicolumn{6}{|l|}{ Medication and Treatment } \\
\hline MT1 medication need & 1.73 & 1.31 & 22.69 & 11.15 & 0.4979 \\
\hline MT2 medication satisfaction & 1.29 & 1.24 & 33.27 & 6.73 & 0.2391 \\
\hline MT3 concerns on side effects & 1.42 & 1.32 & 33.27 & 10 & 0.4178 \\
\hline MT4 frustration on standard medication & 2.03 & 1.47 & 22.31 & 22.12 & 0.5724 \\
\hline MT5 limitation from medication use & 0.77 & 1.03 & 54.81 & 2.31 & 0.5339 \\
\hline MT6 frustration on no disease cure & 2.72 & 1.25 & 5 & 36.92 & 0.5648 \\
\hline \multicolumn{6}{|l|}{ Social and Emotional } \\
\hline SE1 depression due to oral disease & 1.75 & 1.17 & 13.85 & 8.65 & 0.798 \\
\hline SE2 anxiety due to oral disease & 1.42 & 1.17 & 25.38 & 5.96 & 0.7025 \\
\hline SE3 stress due to oral disease & 1.51 & 1.24 & 24.23 & 9.23 & 0.7569 \\
\hline SE4 frustration on disease unpredictability & 1.97 & 1.22 & 11.54 & 12.69 & 0.7212 \\
\hline SE5 worries about the future & 2.08 & 1.33 & 13.85 & 19.04 & 0.3687 \\
\hline SE6 pessimism about the future & 1.27 & 1.25 & 36.35 & 6.35 & 0.5989 \\
\hline SE7 social disruption & 1.12 & 1.22 & 42.12 & 5.96 & 0.7152 \\
\hline \multicolumn{6}{|l|}{ Patient Support } \\
\hline PS1 satisfaction on available information & 1.38 & 1.01 & 20.96 & 2.31 & 0.2857 \\
\hline PS2 support from family & 1.22 & 1.14 & 32.31 & 4.42 & 0.2212 \\
\hline PS3 support from friends/colleagues & 1.42 & 1.27 & 30.96 & 8.65 & 0.2493 \\
\hline PS4 isolation due to oral disease & 0.84 & 1.15 & 55.96 & 4.04 & 0.6407 \\
\hline
\end{tabular}

Table 4 Factor loadings of the remaining 15 COMDQ items using exploratory factor analysis with Promax rotation $(\mathrm{N}=260)$

\begin{tabular}{|l|c|c|c|c|}
\hline \multirow{2}{*}{ Item } & \multicolumn{4}{|c|}{ Extracted factors } \\
\cline { 2 - 5 } & $\begin{array}{c}\text { Physical } \\
\text { Discomfort }\end{array}$ & $\begin{array}{c}\text { Medication \& } \\
\text { Treatment }\end{array}$ & $\begin{array}{c}\text { Social \& } \\
\text { Emotional }\end{array}$ & $\begin{array}{c}\text { Patient } \\
\text { Support }\end{array}$ \\
\hline PF1 discomfort/food types & $\mathbf{0 . 6 2 5}$ & 0.087 & 0.004 & 0.024 \\
\hline
\end{tabular}

This article is protected by copyright. All rights reserved 


\begin{tabular}{|l|r|r|r|r|} 
PF3 discomfort/food texture & $\mathbf{0 . 8 3 6}$ & -0.067 & 0.02 & -0.014 \\
PF5 discomfort/food temperature & $\mathbf{0 . 7 2 8}$ & -0.012 & -0.013 & -0.009 \\
PF7 discomfort/oral hygiene care & $\mathbf{0 . 8 3}$ & 0.018 & 0.009 & -0.068 \\
MT1 medication need & $\mathbf{0 . 3 6 9}$ & 0.151 & 0.148 & 0.025 \\
MT3 concerns on side effects & -0.085 & $\mathbf{0 . 7 9 4}$ & -0.079 & 0.012 \\
MT4 frustration on standard medication & 0.049 & $\mathbf{0 . 6 2 5}$ & 0.098 & 0.081 \\
MT5 limitation from medication use & 0.086 & $\mathbf{0 . 6 5 4}$ & 0.091 & -0.044 \\
SE1 depression due to oral disease & 0.237 & -0.08 & $\mathbf{0 . 8 0 5}$ & 0.097 \\
SE2 anxiety due to oral disease & -0.057 & 0.034 & $\mathbf{0 . 8 9 7}$ & 0.066 \\
SE4 frustration on disease unpredictability & 0.141 & 0.054 & $\mathbf{0 . 7 0 7}$ & -0.01 \\
SE6 pessimism about the future & -0.076 & 0.245 & $\mathbf{0 . 6 8}$ & -0.18 \\
SE7 social disruption & 0.247 & 0.05 & $\mathbf{0 . 5 8 4}$ & 0.058 \\
PS2 support from family & 0.032 & 0.107 & -0.104 & $\mathbf{0 . 6 7 4}$ \\
PS3 support from friends/colleagues & -0.071 & -0.056 & 0.131 & $\mathbf{0 . 8}$ \\
\hline Eigenvalues & 6.707 & 1.468 & 1.427 & 1.027 \\
\hline
\end{tabular}

Note: Factor loadings greater than 0.3 in bold

Table 5 Fit indices summary of the 4-factor solution of the COMDQ-15 and its original scale

\begin{tabular}{|l|c|c|c|c|}
\hline & RMSEA & SRMR & CFI & TLI \\
\hline 15-item COMDQ & 0.08 & 0.04 & 0.97 & 0.97 \\
\hline
\end{tabular}

This article is protected by copyright. All rights reserved 
Table 6 Spearman's rank correlation coefficients between the subscale and total scores of the COMDQ-15 and their corresponding subscale and total score of the full version $(\mathrm{N}=260)$

\begin{tabular}{|l|c|c|c|c|c|}
\hline \multirow{2}{*}{ COMDQ-15 } & \multicolumn{4}{|c|}{ The original COMDQ } \\
\cline { 2 - 5 } & $\begin{array}{c}\text { Pain\& Functional } \\
\text { limitation }\end{array}$ & $\begin{array}{c}\text { Medication \& } \\
\text { Treatment }\end{array}$ & $\begin{array}{c}\text { Social \& } \\
\text { Emotional }\end{array}$ & $\begin{array}{c}\text { Patient } \\
\text { Support }\end{array}$ & Total score \\
\hline $\begin{array}{l}\text { Physical Discomfort } \\
\text { Medication \& Treatment } \\
\text { Social \& Emotional }\end{array}$ & $0.96^{*}$ & $0.93^{*}$ & & & \\
\hline
\end{tabular}


${ }^{*}$ All correlation coefficients were statistically significant with $\mathrm{P}<0.01$ 